\title{
On the Maunder Type Theorems in the Ex-homotopy Category
}

By

\author{
Yoshimi SHITANDA*
}

\begin{abstract}
We study the homotopy theory of comma category and define the cell structure and Postnikov system in the ex-homotopy category. By using these structures we give the four types of spectral sequences and show that Maunder type theorems hold for these spectral sequences.
\end{abstract}

\section{Introduction}

In [5], C. R.F. Maunder defined the cohomology spectral sequence associated with the Postnikov decomposition of $\Omega$-spectrum of target object and showed that his spectral sequence coincides with Atiyah-Hirzebruch spectral sequence. In [4], T. Matumoto proved Maunder's theorem in the equivariant homotopy category. We remarked in [7] that Maunder's theorem holds also in the category of functor complexes. In [6], we studied the unstable version of Maunder's theorem and applied them to the theory of phantom maps. Thus it is interesting to know whether Maunder type theorem holds in a homotopy category. In this paper, we define homotopy spectral sequences associated with cell structure and Postnikov system and prove Maunder type theorems in the ex-homotopy category.

In $\S 1$, we study the homotopy theory of comma category and obtain results analogous to the ones of the ordinary homotopy theory (e. g. J. H. C. Whitehead's theorem). In $\S 2$, we define the cell structure and Postnikov system in the ex-homotopy category and obtain the duality between them. In $\S 3$, we define the homotopy spectral sequences associated with the cell decomposition of a source object and the Postnikov decomposition of a target object by the same way as [6]. In this paper, we shall show that these homotopy spectral sequences are isomorphic as exact couples. Moreover analogously we define the homotopy spectral sequences associated with the anti-skeleton filtration and anti-Postnikov decomposition defined in $\S 3$. We also prove that these are isomorphic as exact couples.

Communicated by N. Shimada, September 14, 1988. Revised December 19, 1988.

* Kurume Institute of Technology, Kurume 830, Japan. 


\section{$\S 1$. The Homotopy Theory of Comma Category}

We review the abstract homotopy theory defined in [7]. In this paper, we shall use the results in [7] and terminologies and notations in S. MacLane [3].

Definition 1.1. We call a category $\mathcal{C}$ a pre-homotopy category if it satisfies the following axioms (A1-3).

(A1) $\mathcal{C}$ is closed under finite limits and finite colimits; hence it has the initial object $\phi$ and the terminal object 1 .

(A2) There are given covariant functors $I, P: \mathcal{C} \rightarrow \mathcal{C}$ with a natural isomorphism $\mathcal{C}(I A, B) \cong \mathcal{C}(A, P B)$ for any objects $A, B$ of $\mathcal{C}(\mathcal{C}(-,-)$ is hom-set in $\mathcal{C}$ ). We call these the cylinder and path functors respectively.

(A3) Moreover there are three natural transformations $k_{*}: I d \rightarrow I(k=0,1)$ and $\tau: I \rightarrow I d$ with $\tau 0_{*}=I d=\tau 1_{*}$. Here $I d$ means the identity functor or identity natural transformation. $0_{*}, 1_{*}$ and $\tau$ are called the top-face, bottom-face and projection transformations respectively.

Let $I^{n}$ be $n$-time composed functor of $I\left(I^{0}=I d\right)$; and define the natural transformations $d_{j}{ }^{k}=I^{n-j} k_{*} I^{j}: I^{n} \rightarrow I^{n+1}$ and $s_{\jmath}=I^{n-j} \tau I^{j}: I^{n+1} \rightarrow I^{n}$ for $(j, k) \in$ $[n] \times[1]([m]=\{0,1, \cdots, m\})$. We call these the face and degeneracy operators respectively. These operators $d_{j}{ }^{k}, s_{j}$ satisfy the cubical simplicial relations (cf. Lemma 1.3 in [7]). Let $\left(i_{0}, k_{0}\right) \in[n] \times[1]$. Then by patching the $2 n+1$ faces $d_{i}{ }^{k}: I^{n} \rightarrow I^{n+1}$ for $(i, k) \neq\left(i_{0}, k_{0}\right)$ according to the cubical simplicial relations, we have the functors $J^{n}=J^{n}\left(i_{0}, k_{0}\right)$ and the natural transformation $\lambda: J^{n} \rightarrow I^{n+1}$. We use the letter $J^{n}$ for any $\left(i_{0}, k_{0}\right)\left(J^{0}=I d\right)$.

Now we consider the following extension condition and the natural homotopy axioms for a pre-homotopy category $\mathcal{C}$ :

(E. C) For any morphism $f: J^{n} X \rightarrow Y$, there is a morphism $F: I^{n+1} X \rightarrow Y$ with $F \lambda=f$.

(NHA 1) There is a natural transformation $\mu: I^{n} \rightarrow J^{n-1}$ with $\mu \lambda=I d$ for all $n>0$, that is, (EC) holds naturally by taking $F \mu=f$.

(NHA 2) There is a natural transformation $\mu: I^{n} \rightarrow J^{n-1}$ with $\tau_{n}^{\prime} \mu=\tau_{n}$ and $\mu \lambda=I d$ for all $n>0$, where $\tau_{n}: I^{n} \rightarrow I d$ and $\tau_{n}^{\prime}: J^{n-1} \rightarrow I d$ are defined by compositions of projections $\tau$.

Let $\mathcal{C}$ be a pre-homotopy category. We call $\mathcal{C}$ an abstract homotopy category if it satisfies (NHA 2). The category CGH of compactly generated Hausdorff spaces and continuous mappings becomes our abstract homotopy category and so does the pointed category $\mathrm{CGH}_{*}$ of $\mathrm{CGH}$ (cf. Example 1.7 in [7]). We say that two morphisms $f_{0}, f_{1}: X \rightarrow Y$ are homotopic (relative $j: A \rightarrow X$ ), if there is a morphism $f: I X \rightarrow Y$ with $f_{k}=f k_{*}$ for $k=0,1$ (and $f I j=f_{0} j \tau$ ); and then we write $f_{0} \simeq f_{1}$ (rel $j$ ) and call $f$ a homotopy of $f_{0}$ and $f_{1}$. When $f, g: I X \rightarrow Y$ are homotopies with $f 1_{*}=g 0_{*}$, we can define a sum $f \oplus g$ of homotopies $f$ and $g$ as usual which is unique up to homotopy relative $\dot{I}=\left\{0_{*} \Perp 1_{*}\right\}$ (the terminal 
faces).

Here we note on the dual considerations. By using the unit $\eta: I d \rightarrow P I$ and the counit $\varepsilon: I P \rightarrow I d$, we have the following axiom (A3*) which is dual and equivalent to (A3) by defining $k^{*}=\varepsilon k_{*} P(k=0,1)$ and $\sigma=P(\tau) \eta$ :

$\left(\mathrm{A} 3^{*}\right)$ There are three natural transformations $k^{*}: P \rightarrow I d(k=0,1)$ and $\sigma: I d \rightarrow P$ with $0 * \sigma=I d=1 * \sigma$, called the top-coface, bottom-coface and injection transformations respectively.

By using $\left(\mathrm{A}^{*}\right)$ and dual constructions, we can obtain the duality principle in our abstract homotopy (cf. [7]).

Let $\mathcal{C}$ be a pre-homotopy category. We say that $j: A \rightarrow X$ in $\mathcal{C}$ (resp. $p: Y \rightarrow B$ ) has the relative $H E P$ (resp. relative $H L P$ ), if any commutative square

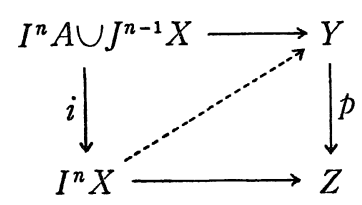

has a dotted morphism to obtain two commutative triangles for any $p: Y \rightarrow B$ with HLP (resp. $j: A \rightarrow X$ with HEP) and all $n>0$. Note: When $Z=1, j$ is called HEP. When $A=\phi, p$ is called HLP. HEP (resp. HLP) is known as homotopy extension (resp. lifting) property, and called a cofibration (resp. fibration) in CGH. Here $X \cup_{A} B$ (abbr. $X \cup B$ ) means the pushout of diagram $X \leftarrow A \rightarrow B$.

We consider the comma calegory $\mathcal{C}_{B}^{A}$ for fixed objects $A, B$ and a fixed morphism $a: A \rightarrow B$ in $\mathcal{C}$ whose objects is any diagram $A \stackrel{x}{\rightarrow} X \stackrel{p}{\rightarrow} B$ in $\mathcal{C}$ with $p x=a$ and whose morphism $f:(A \stackrel{x}{\rightarrow} X \stackrel{p}{\rightarrow} B) \rightarrow(A \stackrel{y}{\rightarrow} Y \stackrel{q}{\rightarrow} B)$ is any morphism $f: X \rightarrow Y$ in $\mathcal{C}$ with $f x=y$ and $q f=p$. For $A \stackrel{x}{\rightarrow} X \stackrel{p}{\rightarrow} B, I, P, k_{*}$ and $\tau$ in $\mathcal{C}$ give us the diagrams
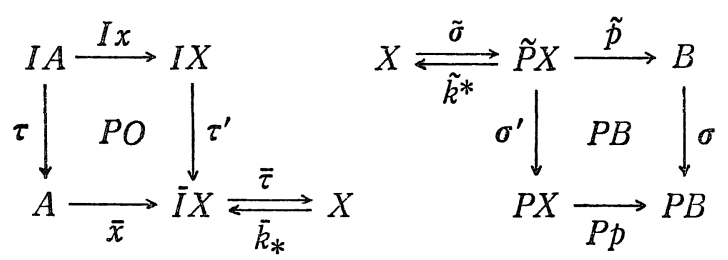

with $\bar{\tau} \bar{x}=x, \bar{\tau} \tau^{\prime}=\tau, \bar{k}_{*}=\tau^{\prime} k_{*}, \tilde{p} \tilde{\sigma}=p, \sigma^{\prime} \tilde{\sigma}=\sigma$ and $\tilde{k}^{*}=k^{*} \sigma^{\prime}$. Hence we have $A \stackrel{\bar{x}}{\rightarrow} \bar{I} X \stackrel{\bar{p}}{\rightarrow} B(\bar{p}=p \bar{\tau})$ and $A \stackrel{\tilde{x}}{\rightarrow} \widetilde{P} X \stackrel{\tilde{p}}{\rightarrow} B(\tilde{x}=\tilde{\sigma} x)$ in $\mathcal{C}_{B}^{A}$, the functors $\tilde{I}, \tilde{P}: \mathcal{C}_{B}^{A} \rightarrow \mathcal{C}_{B}^{A}$ and the natural transformations $\bar{\tau}: \bar{I} \rightarrow I d, \bar{k}_{*}: I d \rightarrow \bar{I}$, satisfying (A2-3). Thus we have a theorem: If $\mathcal{C}$ is a pre-homotopy category, then so is the comma category $\mathcal{C}_{B}^{A}$. Moreover if $\mathcal{C}$ satisfies NHA 2, then so does the comma category $\mathcal{C}_{B}^{A}$ (cf. Theorem 1.9 in $[7]$ ). 
When $B=1$ (resp. $A=\phi$ ), we write simply $(A \rightarrow X)$ (resp. $(X \rightarrow B)$ ) for any object in $\mathcal{C}_{1}^{A}$ (resp. $\mathcal{C}_{B}^{\phi}$ ). When $A=B$ and $p x=i d_{B}$, this comma category is called the ex-homotopy category (cf. [2]) and noted by $\mathcal{C}_{B}$. This category has the zero object $(B \stackrel{i d}{\longrightarrow} B \stackrel{i d}{\longrightarrow} B)$. We write $\mathcal{C}_{B}^{A}[-;-]$ (resp. $\left.\mathcal{C}_{B}[-;-]\right)$ for the homotopy set in $\mathcal{C}_{B}^{A}$ (resp. $\mathcal{C}_{B}$ ).

In our abstract homotopy category $\mathcal{C}$, mapping cylinder $M(f)$, cone $C X$ and suspension $\Sigma X$ are defined by pushouts of diagrams $I X \stackrel{1 *}{\leftarrow}{ }^{f} \rightarrow Y, I X \stackrel{1 *}{\leftarrow} X \rightarrow 1$ and $C X \leftarrow X \rightarrow 1$ respectively. D. Puppe's theorem (Theorem III.6.11 in [8]) and the homotopical invariance of induced (co)fibrations etc. hold also in $\mathcal{C}$ (cf. $[7 ; \S 2]$ ). Note that the suspension functor $\Sigma_{B}$ has the right adjoint functor $\Omega_{B}$ (loop functor) in the ex-homotopy category, because it has the zero object. Generally this fact is not true for $p x \neq i d_{B}$.

The following result is well-known, but we give a proof under our abstract homotopy theory (cf. [1, 8]).

Lemma 1.2. Let $\mathcal{C}$ be a pre-homotopy category satisfying (EC) and $A$ a fixed object in $\mathcal{C}$. For a given morphism $f:(A \stackrel{x}{\rightarrow} X) \rightarrow(A \stackrel{y}{\rightarrow} Y)$ in $\mathcal{C}_{1}^{A}$ where $x$ and $y$ have HEP, if $f: X \rightarrow Y$ is a homotopy equivalence in $\mathcal{C}$, then so is $f$ in $\mathcal{C}_{1}^{A}$.

Proof. By assumptions, there is a homotopy inverse $g: Y \rightarrow X$ of $f$ in $\mathcal{C}$ with $g y=x$. Let $H$ (resp. $K$ ) be a homotopy of $I d$ and $g f$ (resp. $I d$ and $f g$ ) and $L: M(y)=I A \cup Y \rightarrow X$ a morphism defined by $H I x: I A \rightarrow X$ and $g: Y \rightarrow X$. Since $M(y)$ is a retract of $I Y$ by $y \in \mathrm{HEP}$, there is a morphism $\bar{L}: I Y \rightarrow X$ which is the composition of the above retraction and $L$. Set $g^{\prime}=\bar{L} 0_{*}$ which satisfies $g^{\prime} y=x$. Let $M=\bar{L} I(f) \oplus H^{-1}: I X \rightarrow X$ be the sum of $\bar{L} I(f)$ and a reverse homotopy $H^{-1}$ of $H$ which is a homotopy of $g^{\prime} f$ and $I d$. Clearly homotopies $M I(x)$ and $x \tau: I A \rightarrow X$ are homotopic relative $\dot{I}$ (i.e. the terminal faces), there is a homotopy $\tilde{M}: I^{2} A \rightarrow X$ of $M I(x)$ and $x \tau$. Since $I x: I A \rightarrow I X$ has HEP, there is $N: I^{2} X \rightarrow X$ with $\tilde{M}=N I^{2} x, N\left(0_{*} I\right)=M, N\left(I 0_{*}\right)=g^{\prime} f \tau$ and $N\left(I 1_{*}\right)=\tau$. Hence $N\left(1_{*} I\right): I X \rightarrow X$ gives us a homotopy relative $x$ of $g^{\prime} f$ and $I d$. Analogously we have $f^{\prime}: X \rightarrow Y$ constructed as above with $f^{\prime} g^{\prime} \simeq I d$ relative $y$. Since $f \simeq\left(f^{\prime} g^{\prime}\right) f=f^{\prime}\left(g^{\prime} f\right) \simeq f^{\prime}($ rel $x)$, then we have $f \simeq f^{\prime}: X \rightarrow Y$ relative $x$. Thus we have the result.

Corollary 1.3. For a given morphism $f:(A \stackrel{x}{\rightarrow} X \stackrel{p}{\rightarrow} B) \rightarrow(A \stackrel{y}{\rightarrow} Y \stackrel{q}{\rightarrow} B)$ in $\mathrm{CGH}_{B}^{A}$ where $x$ and $y$ have HEP (i.e. NDR pairs in CGH) and $p$ and $q$ have $\operatorname{HLP}(i . e$. fibration in $\mathrm{CGH}$ ), if $f$ is a homotopy equivalence in $\mathrm{CGH}$, then so is $f$ in $\mathrm{CGH}_{B}^{A}$.

Proof. By the above lemma, $f$ is a homotopy equivalence in $\mathrm{CGH}_{1}^{A}$. By the covering homotopy extension theorem (cf. Theorem I.7.16 in [8]) $p:(A \stackrel{x}{\rightarrow} X) \rightarrow(A \stackrel{p x}{\rightarrow} B)$ and $q:(A \stackrel{y}{\rightarrow} Y) \rightarrow(A \stackrel{q y}{\rightarrow} B)$ have HLP in $\mathrm{CGH}_{B}^{A}$. Hence by the 
dual one of the above lemma, $f$ is a homotopy equivalence in $\mathrm{CGH}_{B}^{A}$.

Proposition 1.4. (Corollary 1.1.8. in [1]) Let $\mathcal{C}$ be a pre-homotopy category satisfying (EC). Consider the commutative diagram in $\mathcal{C}$ where $(A \stackrel{x}{\rightarrow} X)$ and $\left(A^{\prime} \stackrel{x^{\prime}}{\rightarrow} X^{\prime}\right)$ have HEP and $(Y \stackrel{q}{\rightarrow} B)$ and $\left(Y^{\prime} \stackrel{q^{\prime}}{\rightarrow} B^{\prime}\right)$ have relative HLP and $h_{i}, k_{i}$ $(i=1,2)$ are homotopy equivalences in $\mathcal{C}$.

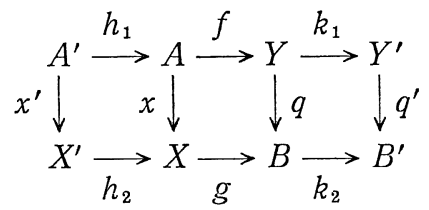

Then there hold the equalities

$$
\mathcal{C}_{B}^{A^{\prime}}\left[X^{\prime} ; Y\right] \cong \mathcal{C}_{B}^{A}[X ; Y] \cong \mathcal{C}_{B^{\prime}}^{A}\left[X ; Y^{\prime}\right] .
$$

Proof. When left-hand square is pushout, the first equality holds by the universality of pushout. Hence we may assume $h_{1}=I d$ by taking the pushout of $A \stackrel{h_{1}}{\leftarrow} A^{\prime} \stackrel{x^{\prime}}{\rightarrow} X$. Since $(A \stackrel{x}{\rightarrow} X)$ and $\left(A^{x^{\prime}} \rightarrow X^{\prime}\right)$ are homotopy equivalent in $\mathcal{C}_{1}^{A}$ by Lemma 1.2 , we can reduce $A=\phi$ by considering in $\mathcal{C}_{1}^{A}$, and may assume $g=i d$ and $q: Y \rightarrow X \in$ HLP by considering the induced fibration through $g$. Then the first equality follows from the homotopical uniqueness of the induced fibrations (cf. Theorem 2.3 in [7]). By the duality and definition of relative HLP, we obtain the second equality.

Proposition 1.5. Let $\mathcal{C}$ be a pre-homotopy category with the zero object satisfying (EC). Consider the diagram where the upper horizontal sequence is a cofiber sequence and the lower one is a fiber sequence and the right-hand square is homotopy comnutative and $\psi^{\wedge}$ is adjoint of $\phi$. Then there exists a morphism $\gamma: B \rightarrow F_{p}$ which makes the diagram homotopy commutative.

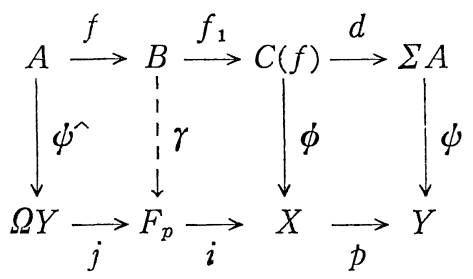

Proof. The above theorem is reduced to the following situation where $f$ (resp. $p$ ) have HEP (resp. HLP) and $F_{p}=p^{-1}(*)$ (i. e. a fiber of $p$ ), $C(f)=B \cup C A$, $\Sigma A=C A / A, p \phi=\phi d$ by the mapping cylinder property (cf. Lemma 2.2 in [7]). Clearly there exist $\gamma: B \rightarrow F_{p}$ with $i \gamma=\phi f_{1}$. Now, we must prove $\gamma f \simeq j \psi^{\wedge}$. Let $\eta$ be adjoint of $\left.\phi\right|_{C A}: C A \rightarrow C(f) \rightarrow X$. Then we can define $\phi^{\prime}=(\eta, \gamma f): A \rightarrow$ 
$L X \times_{X} F_{p}=L X \times_{Y} *$ which is homotopy equivalent to $\psi^{\wedge}: A \rightarrow L Y \times_{Y} *$ by composing $L X \times_{X} F_{p} \stackrel{\simeq}{\longrightarrow} Y \times_{Y^{*}}$. By the commutativity $\gamma f=j^{\prime} \psi^{\prime}$ where $j^{\prime}: L X \times_{X} F_{p}$ $\rightarrow F_{p}$ and $j: L Y \times_{I^{*} * \longleftarrow} \stackrel{\simeq}{\simeq} L \times_{X} F_{p} \rightarrow F_{p}$, we have the result.

Defimition 1.6. (1) The connectivity of $f: X \rightarrow Y$ in $\mathrm{CGH}$ is the maximal integer in $\left\{n ; \pi_{i}(M(f), X)=0\right.$ for $\left.0 \leqq i \leqq n\right\}$.

(2) The coconnectivity of $f: X \rightarrow Y$ in $\mathrm{CGH}$ is the minimal integer in $\left\{n ; \pi_{\imath}(M(f), X)=0\right.$ for $\left.i \geqq n\right\}$.

Lemma 1.7. Consider the commutative diagram (*) in CGH where $(X, A)$ is a relative $\mathrm{CW}$ complex and $p$ is a fibration, and $p=q j$ is the factorization of mapping cylinder with $q k=I d$.

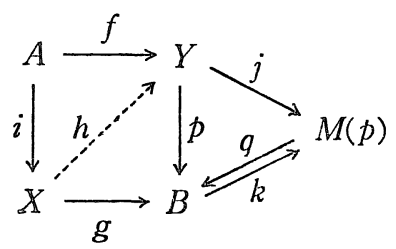

If $\mathrm{kg}$ deform into $Y$ relaiive $A(e . g \cdot \operatorname{dim}(X, A) \leqq$ connectivity of $p$ or connectivity of $(X, A) \geqq($ coconnectivity of $p)-1)$, then there exists $h: X \rightarrow Y$ with $h i=f$ and $p h=g$.

Proof. Since a homotopy $H$ of $k g i$ and $j f$ can be constructed by using the cylinder $I Y$ of $Y$, there is $\bar{H}: I X \rightarrow M(p)$ with $H=\bar{H} I(i)$ and $\bar{H} 0_{*}=k g$ by $i \in \mathrm{HEP}$. Thus we have $\bar{g}=\bar{H} 1_{*}: X \rightarrow M(p)$ with $\bar{g} i=j f$ and $k g \simeq \bar{g}$. Hence $g$ and $q \bar{g}$ are homotopic relative $A$. By assumptions, there is $\tilde{g}: X \rightarrow Y$ with $\tilde{g} i=f$ and $j \tilde{g} \simeq \bar{g}$ relative $i$. Therefore $p \tilde{g}$ and $g$ are homotopic relative $A$ by $p \tilde{g}=q j \tilde{g} \simeq q \bar{g}$ (rel $i$ ) $\simeq q k g=g($ rel $i)$. By the covering homotopy extension theorem, we can choose $h: X \rightarrow Y$ with $h i=f$ and $p h=g$ 。

Propcsition 1.8. Let $f:\left(A \stackrel{x}{\rightarrow} X^{\stackrel{p}{\rightarrow}} B\right) \rightarrow\left(A \stackrel{y}{\rightarrow} Y^{q} \rightarrow B\right)$ be a morphism in $\mathrm{CGH}_{B}^{A}$ where $p$ and $q$ are fibrations and $f$ is n-connected in $\mathrm{CGH}$, and $(A \stackrel{\vec{z}}{\rightarrow} \underset{\stackrel{r}{\rightarrow}}{\rightarrow} B)$ an object in $\mathrm{CGH}_{B}^{A}$ where $(Z, A)$ is a relative $\mathrm{CW}$ complex. Then the induced map

(1) $f_{*}: \mathrm{CGH}_{B}^{A}[Z ; X] \rightarrow \mathrm{CGH}_{B}^{A}[Z ; Y]$ is bijective, if $\operatorname{dim}(Z, A) \leqq n-1$.

(2) $f_{*}: \mathrm{CGH}_{B}^{A}[Z ; X] \rightarrow \mathrm{CGH}_{B}^{A}[Z ; Y]$ is surjecive, if $\operatorname{dim}(Z, A) \leqq n$.

Proof. We factorize $f$ as $f=g h: X \stackrel{h}{\rightarrow} X^{\prime} \stackrel{g}{\rightarrow} Y$ where $g$ is a fibration in CGH and $h$ is a homotopy equivalence in CGH. For $h:(A \stackrel{x}{\rightarrow} X \stackrel{p}{\rightarrow} B) \rightarrow\left(A \stackrel{h x}{\longrightarrow} X^{\prime} \stackrel{q g}{\longrightarrow} B\right)$, Proposition 1.8 is true by Proposition 1.4. Hence we may assume $f$ is a fibration in CGH and apply Lemma 1.7 for $A \stackrel{z}{\rightarrow} Z$ and $X \stackrel{f}{\rightarrow} Y$. Thus we obtain the result. 
Proposition 1.9. Let $f:(A \stackrel{x}{\rightarrow} X \stackrel{p}{\rightarrow} B) \rightarrow(A \stackrel{y}{\rightarrow} Y \stackrel{q}{\rightarrow} B)$ be a morphism in $\mathrm{CGH}_{B}^{A}$ where $p$ and $q$ are fibrations and $f$ is $n$-coconnected in $\mathrm{CGH}$, and $(A \stackrel{z}{\rightarrow} Z \stackrel{r}{\rightarrow} B)$ an object in $\mathrm{CGH}_{B}^{A}$ where $(Z, A)$ is a relative $\mathrm{CW}$ complex and c-connected. Then the induced map

(1) $f_{*}: \mathrm{CGH}_{B}^{A}[Z ; X] \rightarrow \mathrm{CGH}_{B}^{A}[Z ; Y]$ is bijective, if $c \geqq n-1$.

(2) $f_{*}: \mathrm{CGH}_{B}^{A}[Z ; X] \rightarrow \mathrm{CGH}_{B}^{A}[Z ; Y]$ is injective, if $c \geqq n-2$.

Proof. We may take $(\bar{Z}, A)$ which is homotopy equivalent relative $A$ to $(Z, A)$ and has no $i$-cells for $0 \leqq i \leqq c$ by the relative $\mathrm{CW}$ approximation theorem. By the same way as Proposition 1.8, we may assume $f$ is a fibration in CGH. Thus we obtain the result by Proposition 1.4 and Lemma 1.7.

Since the proofs of the following propositions are analogous to the ones above, hence we omit them.

Proposition 1.10. Let $f:(A \stackrel{x}{\rightarrow} X \stackrel{p}{\rightarrow} B) \rightarrow(A \stackrel{y}{\rightarrow} Y \stackrel{q}{\rightarrow} B)$ be a morphism in $\mathrm{CGH}_{B}^{A}$ where $(X, A)$ and $(Y, A)$ are relative $\mathrm{CW}$ complexes and $f$ is n-connected in $\mathrm{CGH}$, and $(A \stackrel{z}{\rightarrow} Z \stackrel{r}{\rightarrow} B)$ an object in $\mathrm{CGH}_{B}^{A}$ where $r$ is a fibration and c-coconnected. Then the induced map

(1) $f^{*}: \mathrm{CGH}_{B}^{A}[Y ; Z] \rightarrow \mathrm{CGH}_{B}^{A}[X ; Z]$ is bijective, if $n \geqq c-1$.

(2) $f^{*}: \mathrm{CGH}_{B}^{A}[Y ; Z] \rightarrow \mathrm{CGH}_{B}^{A}[X ; Z]$ is injective, if $n \geqq c-2$.

Proposition 1.11. Let $f:(A \stackrel{x}{\rightarrow} X \stackrel{p}{\rightarrow} B) \rightarrow(A \stackrel{y}{\rightarrow} Y \stackrel{q}{\rightarrow} B)$ be a morphism in $\mathrm{CGH}_{B}^{A}$ where $(X, A)$ and $(Y, A)$ are relative $\mathrm{CW}$ complexes and $f$ is n-dimensional (i.e. $\operatorname{dim}(M(f), X) \leqq n)$ in $\mathrm{CGH}$, and $(A \stackrel{z}{\rightarrow} Z \stackrel{r}{\rightarrow} B)$ an object in $\mathrm{CGH}_{B}^{A}$ where $r$ is a fibration and c-connected. Then the induced map

(1) $f^{*}: \mathrm{CGH}_{B}^{A}[Y ; Z] \rightarrow \mathrm{CGH}_{B}^{A}[X ; Z]$ is bijective, if $n \leqq c-1$.

(2) $f^{*}: \mathrm{CGH}_{B}^{A}[Y ; Z] \rightarrow \mathrm{CGH}_{B}^{A}[X ; Z]$ is surjective, if $n \leqq c$.

\section{§ 2. Cell Structure and Postnikov System}

Definition 2.1. (1) An object $(A \stackrel{x}{\rightarrow} X \stackrel{p}{\rightarrow} B)$ in $\mathrm{CGH}_{B}^{A}$ is called a cell complex in $\mathrm{CGH}_{B}^{A}$, if $A \stackrel{x}{\rightarrow} X$ is a relative $\mathrm{CW}$ complex. The $n$-skeleton of $(A \stackrel{x}{\rightarrow} X \stackrel{p}{\rightarrow} B)$ is defined by the restriction $\left(A \stackrel{x_{n}}{\longrightarrow} X^{n} \stackrel{p_{n}}{\longrightarrow} B\right)\left(x_{n}: A \rightarrow X^{n}, j_{n}: X^{n} \rightarrow X, j_{n} x_{n}=x\right.$, $\left.p j_{n}=p_{n}\right)$ on the $n$-skeleton $X^{n}$ of $X$.

(2) Let $(A \stackrel{x}{\rightarrow} X \stackrel{p}{\rightarrow} B)$ be a cell complex in $\mathrm{CGH}_{B}^{A}$. Then $\left(A \stackrel{x_{m}^{n}}{\longrightarrow} X_{m}^{n} \stackrel{p_{m}^{n}}{\longrightarrow} B\right)$ $=\left(A \stackrel{J_{m}^{n} x_{n}}{\longrightarrow} X^{n} / X^{m} \stackrel{p_{m}^{n}}{\longrightarrow} B\right)$ is defined by the pushout diagram $(0 \leqq m<n \leqq \infty)$ 


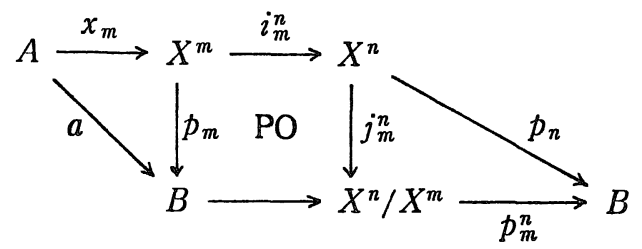

In particular, $i_{n-1}^{n}$ (resp. $\left.j_{n-1}^{n}\right)$ is abbreviated as $i^{n}$ (resp. $\left.j^{n}\right) .\left(X^{n} / X^{m}, B\right)$ is a relative CW complex with $i$-cells for $m<i \leqq n$ and $\left(X^{n} / X^{n-1}, B\right)$ is a relative CW complex with $n$-cells $\left(B \cup_{j} e_{\jmath}^{n}, B\right)$, where $X^{n} / X^{n-1}$ is homotopy equivalent to a wedge $\operatorname{sum} B \vee_{j} e_{j}^{n}$ in $\mathrm{CGH}$.

Remark 2.2. The cone $C(B \rightarrow Z \rightarrow B)=\left(B \rightarrow C_{B} Z \rightarrow B\right)$ of $(B \rightarrow Z \rightarrow B)$ is homotopy equivalent to $(B \stackrel{i d}{\longrightarrow} B \stackrel{i d}{\longrightarrow} B)$ by Lemma 2.2 in [7], in particular $C_{B} Z$ is homotopy equivalent to $B$ in $\mathrm{CGH}$. Hence $X^{n} / X^{m}$ is homotopy equivalent to $X^{n} \cup C_{B} X^{m}$ in $\mathrm{CGH}$ by Theorem 2.5 in [7]. For the classification problem in Proposition 1.4 (when $A=B$ ), we may replace $\left(B \rightarrow X^{n} / X^{m}\right)$ by $\left(B \rightarrow X^{n} \cup C_{B} X^{m}\right)$ in the left-hand side.

Theorem 2.3. (Theorem 6.4 in [8]). Let $q: Y \rightarrow B$ be a fibration with $a$ connected fiber $F$. If $\pi_{1}(Y)$ acts simply on $\pi_{n}(M(q), Y), q$ admits a principal Postnikov system.

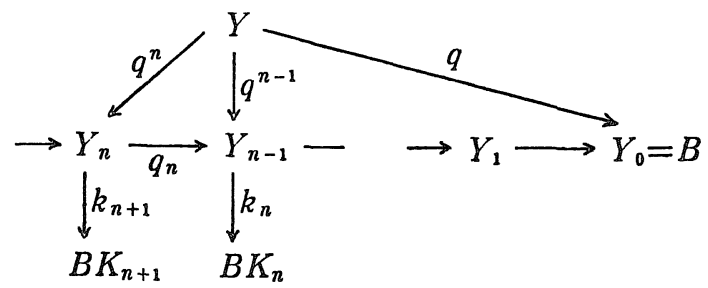

where $q_{n}: Y_{n} \rightarrow Y_{n-1}$ is a fibration induced by $k_{n}: Y_{n-1} \rightarrow K\left(\pi_{n}(F), n+1\right)$ from the siandard path fibration on $B K_{n}\left(K_{n}=K\left(\pi_{n}(F), n\right), B K_{n}=K\left(\pi_{n}(F), n+1\right)\right)$, and $q^{n}: Y \rightarrow Y_{n}$ is $(n+1)$-connected.

By using a fibration $q_{n}: Y_{n} \rightarrow Y_{n-1}$ and induction on $Y_{n}, \pi_{i}\left(Y_{n}\right)$ is equal to $\pi_{i}(Y)$ for $i \leqq n$ and $\pi_{i}(B)$ for $i \geqq n+2$ and $q_{1} \cdots q_{n}: Y_{n} \rightarrow B$ is $(n+2)$-coconnected. Moreover there is an exact sequence

$$
0 \longrightarrow \pi_{n+1}\left(Y_{n}\right) \longrightarrow \pi_{n+1}(B) \longrightarrow \pi_{n}(F) \longrightarrow \pi_{n}\left(Y_{n}\right) \longrightarrow \pi_{n}\left(Y_{n-1}\right) \longrightarrow 0 .
$$

Proposition 2.4. Let $(A \stackrel{x}{\rightarrow} X \stackrel{p}{\rightarrow} B)$ be a cell complex in $\mathrm{CGH}_{B}^{A}$ and $(A \stackrel{y}{\rightarrow} Y \stackrel{q}{\rightarrow} B)$ an object in $\mathrm{CGH}_{B}^{A}$ where $q$ admit a principal Postnikov system and $q_{n}: Y_{n} \rightarrow B$ the Postnikov n-stage of $q$. Then there holds a natural isomorphim:

$$
\mathrm{CGH}_{B}^{A}\left[X ; Y_{n}\right] \cong \text { Image of } \mathrm{CGH}_{B}^{A}\left[X^{n+1} ; Y\right] \longrightarrow \mathrm{CGH}_{B}^{A}\left[X^{n} ; Y\right] \text {. }
$$


Proof. Consider the commutative diagram

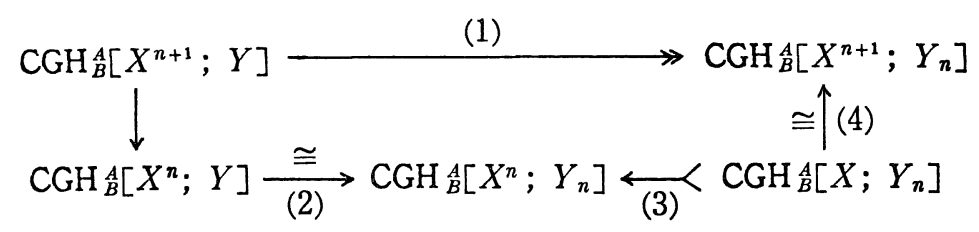

Since $q_{n}: Y \rightarrow Y_{n}$ is $(n+1)$-connected and dimension of $\left(X^{m}, A\right)$ is $m(m=n$, $n+1$ ), (1) is surjective and (2) is isomorphic by Proposition 1.8. Since $q_{1} \cdots q_{n}$ : $Y_{n} \rightarrow B$ is $(n+2)$-coconnected and $\left(X, X^{m}\right)$ is m-connected, (3) is injective and (4) is isomorphic by Proposition 1.10 .

If $q$ has a cross section, $\pi_{i}\left(Y_{n}\right)$ is isomorphic to $\pi_{i}(Y)$ for $i \leqq n$ and $\pi_{i}(B)$ for $i \geqq n+1$, and there holds an exact sequence $0 \rightarrow \pi_{n}(F) \rightarrow \pi_{n}(Y) \rightarrow \pi_{n}(B) \rightarrow 0$.

Since an Eilenberg-MacLane space $K(\pi, n)(\pi$; abelian group, $n \geqq 1)$ can be considered as a topological abelian group, $(B \stackrel{\langle i d, 0\rangle}{\longrightarrow} B \times K(\pi, n) \stackrel{p r}{\longrightarrow} B)$ is homotopy equivalent to $(B \stackrel{\langle i d, r\rangle}{\longrightarrow} B \times K(\pi, n) \stackrel{p r}{\longrightarrow} B)\left(r: B \rightarrow K(\pi, n)\right.$ a continuous map) in $\mathrm{CGH}_{B}$ by using $h: B \times K(\pi, n) \rightarrow B \times K(\pi, n)$ defined by $h(b, x)=(b, x+r(b))$.

Definition 2.5. Let $(B \stackrel{y}{\rightarrow} Y \stackrel{q}{\rightarrow} B)$ be an object in $\mathrm{CGH}_{B}$ where $q$ is a fibration with a connected fiber $F$ and admit a principal Postnikov system.

(1) An object $(B \stackrel{\langle i d, r\rangle}{\longrightarrow} B \times K(\pi, n) \stackrel{p r}{\longrightarrow} B)$ is called an Eilenberg-MacLane object in $\mathrm{CGH}_{B}$.

(2) $k_{n+1}=\left\langle q_{1} \cdots q_{n}, k_{n+1}\right\rangle:\left(B \stackrel{q^{n} y}{\longrightarrow} Y_{n} \stackrel{q_{1} \cdots q}{\longrightarrow} B\right) \rightarrow\left(B \stackrel{\langle i d, r\rangle}{\longrightarrow} B \times B K_{n+1} \stackrel{p r}{\longrightarrow} B\right)$ is called the $k$-invariant of $(B \stackrel{y}{\rightarrow} Y \stackrel{q}{\rightarrow} B)$ in $\mathrm{CGH}_{B}$.

(3) Let $L K_{n} \rightarrow B K_{n}$ be a standard path fibration with a fiber $K_{n}$. Then $\left(B \stackrel{\langle i d, 0\rangle}{\longrightarrow} B \times L K_{n} \stackrel{p r}{\longrightarrow} B\right) \rightarrow\left(B \stackrel{\langle i d, 0\rangle}{\longrightarrow} B \times B K_{n} \stackrel{p r}{\longrightarrow} B\right)$ becomes the standard path fibration with a fiber $\left(B \stackrel{\langle i d, 0\rangle}{\longrightarrow} B \times K_{n} \stackrel{p r}{\longrightarrow} B\right)$ in $\mathrm{CGH}_{B}$.

(4) An object $\left(B \stackrel{q^{n} y}{\longrightarrow} Y_{n} \stackrel{q_{1} \cdots q_{n}}{\longrightarrow} B\right)$ is called the Postnikov $n$-stage of $(B \stackrel{y}{\rightarrow} Y \stackrel{q}{\rightarrow} B)$ in $\mathrm{CGH}_{B}$. There are morphisms $q_{n}:\left(B \stackrel{q^{n} y}{\longrightarrow} Y_{n} \stackrel{q_{1} \cdots q_{n}}{\longrightarrow} B\right) \rightarrow\left(B \stackrel{q^{n-1} y}{\longrightarrow} Y_{n-1} \stackrel{q_{1} \cdots q_{n-1}}{\longrightarrow} B\right)$ and $q^{n}:(B \stackrel{y}{\rightarrow} Y \stackrel{q}{\rightarrow} B) \rightarrow\left(B \stackrel{q^{n} y}{\longrightarrow} Y_{n} \stackrel{q_{1} \cdots q_{n}}{\longrightarrow} B\right)$.

(5) The homotopy fiber in $\mathrm{CGH}_{B}$ of $q^{n}:(B \stackrel{y}{\rightarrow} Y \stackrel{q}{\rightarrow} B) \rightarrow\left(B \stackrel{q^{n} y}{\longrightarrow} Y_{n} \stackrel{q_{1} \cdots q_{n}}{\longrightarrow} B\right)$ defines $\left(B \stackrel{y^{[n+1]} \longrightarrow}{\longrightarrow} Y^{(n+1)} \stackrel{q^{[n+1]}}{\longrightarrow} B\right)$ where $\pi_{\imath}\left(Y^{(n+1)}\right)$ is isomorphic to $\pi_{\imath}(B)$ for $i \leqq n$ and $\pi_{\imath}(Y)$ for $i \geqq n+1$. Note that $\mathrm{CGH}_{B}$ has the zero object $(B \stackrel{i d}{\longrightarrow} B \stackrel{i d}{\longrightarrow} B)$.

(6) $\left(B \stackrel{y_{m}^{n}}{\longrightarrow} Y(m, n) \stackrel{q_{m}^{n}}{\longrightarrow} B\right)$ is defined by the homotopy fiber of $q_{m} \cdots q_{n}$ :

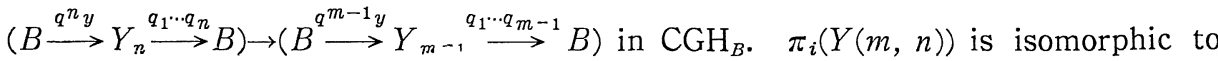


$\pi_{i}(Y)$ for $m \leqq i \leqq n$ and $\pi_{i}(B)$ for $i<m$ or $n<i$. We interpret $Y(-\infty, n)$, $Y(n, \infty)$ as $Y_{n}, Y^{(n)}$ respectively. Clearly $\left(\Omega_{B} Y\right)(m, n)$ is homotopy equivalent to $\Omega_{B}(Y(m+1, n+1))$ and $\left(B \rightarrow \Omega_{B}(B \times K) \rightarrow B\right)$ is homotopy equivalent to $(B \rightarrow B \times \Omega K \rightarrow B)$ in $\mathrm{CGH}_{B}$.

Proposition 2.6. Let $(B \stackrel{x}{\rightarrow} X \stackrel{p}{\rightarrow} B)$ be a cell complex in $\mathrm{CGH}_{B}$ and $(B \stackrel{y}{\rightarrow} Y \stackrel{q}{\rightarrow} B)$ an object in $\mathrm{CGH}_{B}$ wbere $q$ is a fibration with a connected fiber $F$ and admit a principal Postnikov system. Then there is a natural isomorphism:

$\mathrm{CGH}_{B}\left[X ; Y^{(n+1)}\right] \cong$ Image of $\mathrm{CGH}_{B}\left[X / X^{n} ; Y\right] \longrightarrow \mathrm{CGH}_{B}\left[X / X^{n-1} ; Y\right]$.

Proof. Consider the commutative diagram

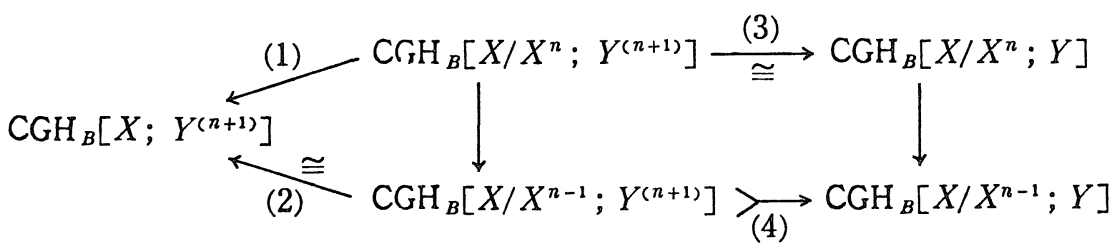

Since $Y^{(n+1)} \rightarrow B$ is $(n+1)$-connected and $X \rightarrow X / X^{m}$ is $m+1$-dimensional $(m=n$, $n-1$ ) (cf. Remark 2.2 and Proposition 1.4.) (1) is surjective and (2) is isomorphic by Proposition 1.11. Since $Y^{(n+1)} \rightarrow Y$ is $(n+1)$-coconnected and $B \rightarrow X / X^{m}$ is $m$-connected $(m=n-1, n),(3)$ is isomorphic and (4) is injective by Proposition 1.9.

Corollary 2.7. Under the assumptions of Proposition 2.6, there is a natural isomorphism :

$\mathrm{CGH}_{B}[X ; Y(m, n)] \cong$ Image of $\mathrm{CGH}_{B}\left[X^{n+1} / X^{m-1} ; Y\right] \longrightarrow \mathrm{CGH}_{B}\left[X^{n} / X^{m-2} ; Y\right]$.

\section{§ 3. Maunder Type Theorems}

In this section we work in the ex-homotopy category $\mathrm{CGH}_{B}$ and assume that all space in $\mathrm{CGH}$ is 0 -connected and has the homotopy type of $\mathrm{CW}$ complex.

Lemma 3.1. Let $\left(B \stackrel{x}{\rightarrow} B \cup e^{n} \stackrel{p}{\rightarrow} B\right)$ be a cell complex in $\mathrm{CGH}_{B}$ and $(B \stackrel{y}{\rightarrow} Y \stackrel{q}{\rightarrow} B)$ an object in $\mathrm{CGH}_{B}$ where $q$ is a fibration with a fiber $F$ and admit a principal Postnikov system. Then $\mathrm{CGH}_{B}\left[B \cup e^{n} ; Y\right]$ is identified with $\pi_{n}(F)$. If $f:\left(B \stackrel{x}{\rightarrow} B \cup e^{n} \stackrel{p}{\rightarrow} B\right) \rightarrow\left(B \stackrel{x^{\prime}}{\rightarrow} B \cup e^{n^{p^{\prime}}} \rightarrow B\right)$ is a map of degree $m$ (i.e. degree of $\left.H_{n}\left(B \cup e^{n}, B\right) \rightarrow H_{n}\left(B \cup e^{n}, B\right)\right)$, then the induces map $f^{*}: \mathrm{CGH}_{B}\left[B \cup e^{n}, Y\right] \rightarrow$ $\mathrm{CGH}_{B}\left[B \cup e^{n}, Y\right]$ is a map of degree $m$.

Proof. The homotopy set $\mathrm{CGH}_{B}\left[B \cup e^{n} ; Y\right]$ is classified by the relative cross sections of the induced fibration $p^{*}(q)$ over $B \cup e^{n}$. Hence it is classified by the relative cross sections of the induced fibration over the $n$-disk $D^{n}$. Since a fibration over $D^{n}$ is fiber homotopy equivalent to the projection $D^{n} \times F \rightarrow D^{n}$ 
and a cross section over $S^{n-1}$ is given, we have the result by assigning the difference cochain $d \in H_{n}\left(D^{n}, S^{n-1} ; \pi_{n} F\right)=\pi_{n} F$. For the second part, let $f:\left(D^{n}, S^{n-1}\right) \rightarrow\left(D^{n}, S^{n-1}\right)$ be a map of degree $m$. Then the induced map of fibrations is fiber homotopy equivalent to $f^{\sim}: D^{n} \times F \rightarrow D^{n} \times F, f^{\sim}(x, y)=(f(x), y)$. Thus $f:\left(D^{n}, S^{n-1}\right) \rightarrow\left(D^{n}, S^{n-1}\right)$ induces a map of multiple $m: \pi_{n}(F) \rightarrow \pi_{n}(F)$ by using the correspondence $\left(D^{n}, S^{n-1}\right) \stackrel{f}{\rightarrow}\left(D^{n}, S^{n-1}\right) \stackrel{s}{\rightarrow} F$ ( $s$ : cross section).

Let $(B \stackrel{x}{\rightarrow} X \stackrel{p}{\rightarrow} B)$ be a cell complex in $\mathrm{CGH}_{B}$ and $(B \stackrel{y}{\rightarrow} Y \stackrel{q}{\rightarrow} B)$ an object in $\mathrm{CGH}_{B}$ where $q$ is a fibration with a fiber $F$ and admit a principal Postnikov system. Hereafter we write simply total space $X$ for an object $(B \stackrel{x}{\rightarrow} X \stackrel{p}{\rightarrow} B)$, unless there happen confusions.

The Puppe sequence in $\mathrm{CGH}_{B}$

$$
X^{s-1} \stackrel{i^{s}}{\longrightarrow} X^{s} \stackrel{j^{s}}{\longrightarrow} X^{s} / X^{s-1} \stackrel{h^{s}}{\longrightarrow} \Sigma_{B} X^{s-1} \longrightarrow \Sigma_{B} X^{s} \longrightarrow \Sigma_{B} X^{s} / X^{s-1} \longrightarrow
$$

gives the exact couple

$$
\begin{aligned}
\left\{{D_{1}}^{s, t}\right. & \left., E_{1}^{s, t}, \alpha_{1}, \beta_{1}, \gamma_{1}\right\} \\
D_{1}{ }^{s, t} & =\mathrm{CGH}_{B}\left[\Sigma_{B}{ }^{t-s} X^{s} ; Y\right] \quad(t \geqq s \geqq 0) \\
E_{1}^{s, t} & =\mathrm{CGH}_{B}\left[\Sigma_{B}{ }^{t-s} X^{s} / X^{s-1} ; Y\right] \\
& =C^{s}\left(X, B ; \pi_{t}(F)\right) \quad(t \geqq s \geqq 0)
\end{aligned}
$$

where $\alpha_{1}, \beta_{1}$ and $\gamma_{1}$ are induced by $i^{s}: X^{s-1} \rightarrow X^{s}, h^{s}: X^{s} / X^{s-1} \rightarrow \Sigma_{B} X^{s-1}, j^{s}: X^{s}$ $\rightarrow X^{s} / X^{s-1}$ respectively. The bidegrees $\alpha_{1}, \beta_{1}$ and $\gamma_{1}$ are $(-1,-1),(1,0)$ and $(0,0)$ respectively. Let $\left\{D_{r}^{s, t}, E_{r}{ }^{s, t}, \alpha_{r}, \beta_{r}, \gamma_{r}\right\}$ be the derived couple of (3.2). Since the differential of (3.2) is induced by $X^{s+1} / X^{s}=B \cup_{i} e_{\imath}^{s+1} \rightarrow \Sigma_{B} X^{s} \rightarrow \Sigma_{B} X^{s} / X^{s-1}$ $=B \cup_{j} e_{j}^{s+1}, E_{2}$-term is described by the following formula in the light of Lemma 3.1.

$$
E_{2}{ }^{s, t}=H^{s}\left(X, B ; \pi_{t}(F)\right) \quad(t \geqq s \geqq 0) .
$$

When $t-s=0, E_{2}$-term is not obtained by $E_{1}$-term. But we may use (3.3) for the $E_{2}$-term by Lemma 3.8 below.

Now let us consider the Postnikov system of $(B \stackrel{y}{\rightarrow} Y \stackrel{q}{\rightarrow} B)$. By using the fiber mapping sequence in $\mathrm{CGH}_{B}$ :

$$
\longrightarrow \Omega_{B} Y_{n-1} \longrightarrow \Omega_{B}\left(B \times B K_{n}\right) \stackrel{l_{n}}{\longrightarrow} Y_{n} \stackrel{q_{n}}{\longrightarrow} Y_{n-1} \stackrel{k_{n}}{\longrightarrow} B \times B K_{n}
$$

we define the exact couple.

$$
\begin{aligned}
& \left\{\bar{D}_{2}^{s, t}, \bar{E}_{2}{ }^{s, t}, \bar{\alpha}_{2}, \bar{\beta}_{2}, \bar{\gamma}_{2}\right\} \\
& \bar{D}_{2}{ }^{s, t}=\mathrm{CGH}_{B}\left[X ; \Omega_{B}{ }^{t-s} Y_{t}\right] \cong \mathrm{CGH}_{B}\left[\Sigma_{B}{ }^{t-s} X ; Y_{t}\right] \quad(t \geqq s \geqq 0) \\
& \bar{E}_{2}{ }^{s, t}=\mathrm{CGH}_{B}\left[X ; \Omega_{B}{ }^{t-s+1}\left(B \times B K_{t}\right)\right] \cong H^{s}\left(X, B ; \pi_{t}(F)\right) \quad(t+1 \geqq s \geqq 0)
\end{aligned}
$$


where $\bar{\alpha}_{2}, \bar{\beta}_{2}$ and $\tilde{\gamma}_{2}$ are induced by $q_{n}: Y_{n} \rightarrow Y_{n-1}, k_{n}: Y_{n-1} \rightarrow B \times B K_{n}$ and $l_{n}: B \times K_{n} \rightarrow Y_{n}$ in $\mathrm{CGH}_{B}$. The bidegrees of $\bar{\alpha}_{2}, \bar{\beta}_{2}$ and $\bar{\gamma}_{2}$ are $(-1,-1),(2,1)$ and $(0,0)$ respectively. Let $\left\{\bar{D}_{r}{ }^{s, t}, \bar{E}_{r}{ }^{s, t}, \bar{\alpha}_{r}, \bar{\beta}_{r}, \bar{\gamma}_{r}\right\}$ be the derived couple of (3.4).

Theorem 3.5. Let $(B \stackrel{x}{\rightarrow} X \stackrel{p}{\rightarrow} B)$ be a cell complex in $\mathrm{CGH}_{B}$ where $(X, B)$ has no 0-cells and $(B \stackrel{y}{\rightarrow} Y \stackrel{q}{\rightarrow} B)$ an object in $\mathrm{CGH}_{B}$ where $q$ is a fibration with a fiber $F$ and admit a principal Postnikov system. Then, for $t-s \geqq 0$, there exist isomorphisms

$$
\phi: D_{r}^{s, t} \longrightarrow \bar{D}_{r}^{s, t}, \quad \phi: E_{r}^{s, t} \longrightarrow \bar{E}_{r}^{s, t}
$$

which commute $\alpha_{r}, \beta_{r}, \gamma_{r}$ and $\bar{\alpha}_{r}, \bar{\beta}_{r}, \bar{\gamma}_{r}$.

The proof of this theorem proceeds by the same way as [6]. Let consider the diagram

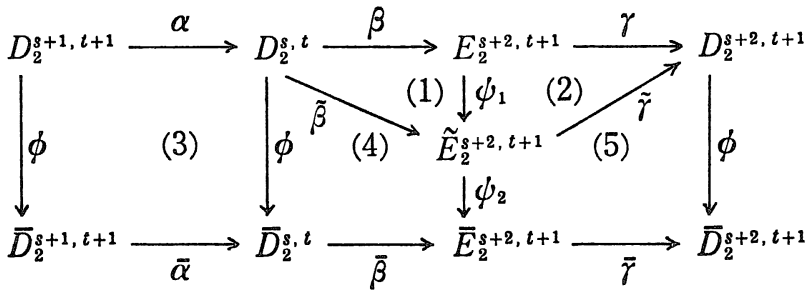

We shall define the group $\tilde{E}_{2}{ }^{s, t}(t-s \geqq 1)$, the isomorphisms $\phi, \phi_{1}, \phi_{2}, \phi_{2} \phi_{1}=\phi$ and homomorphisms $\tilde{\beta}_{2}$ and $\tilde{\gamma}_{2}$ which make the diagram commutative.

Let $Y^{(k)}$ be the homotopy fiber in $\mathrm{CGH}_{B}$ of $q_{k-1}: Y \rightarrow Y_{k-1}, i^{(t)}: Y^{(t)} \rightarrow Y$ the canonical inclusion and $q^{(t)}: Y^{(t)} \rightarrow B \times K_{t}$ the natural morphism in $\mathrm{CGH}_{B}$. By the proof of Proposition 2.4, $q_{t}$ induces an isomorphism (cf. [6]):

$$
\phi: D_{2}^{s, t} \longrightarrow \bar{D}_{2}^{s, t} \text { is an isomorphism for } t-s \geqq 0 \text {. }
$$

We shall define $\tilde{E}_{2}{ }^{s, t}$ for $t-s \geqq 0$.

$$
\begin{aligned}
\tilde{E}_{2}^{s, t} & =\text { Image of } \mathrm{CGH}_{B}\left[\Sigma_{B}^{t-s} X^{s+1} ; Y^{(t)}\right] \longrightarrow \mathrm{CGH}_{B}\left[\Sigma_{B}{ }^{t-s} X^{s} ; Y^{(t)}\right] \text { for } t-s \geqq 0 \\
& =\operatorname{Ker} \text { of } h^{s+1} *: \mathrm{CGH}_{B}\left[\Sigma_{B}^{t-s} X^{s} ; Y^{(t)}\right] \longrightarrow \mathrm{CGH}_{B}\left[\Sigma_{B}{ }^{t-s-1} X^{s+1} / X^{s} ; Y^{(t)}\right] \\
& \text { for } t-s \geqq 1 .
\end{aligned}
$$

Lemma 3.8。 (1) There exists a natural isomorphism $\phi_{2}: \widetilde{E}_{2}{ }^{s, t} \rightarrow \bar{E}_{2}{ }^{s, t}$ for $t-s \geqq 0$.

(2) The map js*: $\mathrm{CGH}_{B}\left[\Sigma_{B}{ }^{t-s} X^{s} / X^{s-1} ; Y^{(t)}\right] \rightarrow \mathrm{CGH}_{B}\left[\Sigma_{B}^{t-s} X^{s} ; Y^{(t)}\right]$ induces an isomorphism $\phi_{1}: E_{2}{ }^{s, t} \rightarrow \tilde{E}_{2}{ }^{s, t}$ for $t-s \geqq 0$.

Proof. (1) is proved by Proposition 2.4. (2) By the following diagram we have the result for $t-s \geqq 1$. 


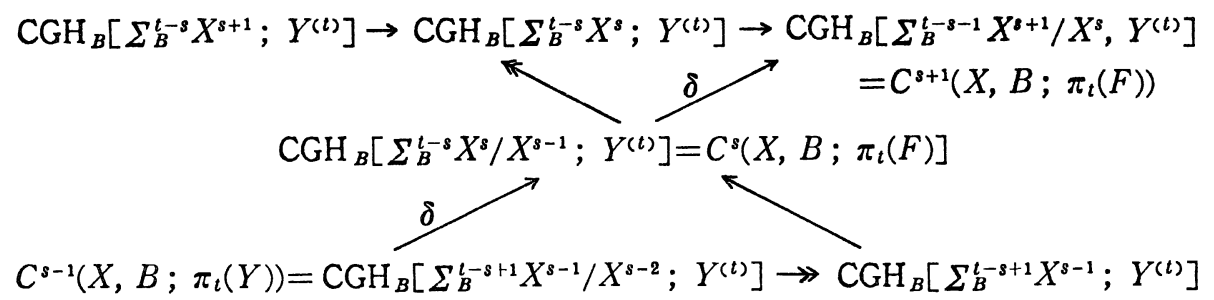

For $t-s=0$, the map $j^{s *}: \mathrm{CGH}_{B}\left[X^{s} / X^{s-1} ; Y^{(s)}\right] \rightarrow \mathrm{CGH}_{B}\left[X^{s} ; Y^{(s)}\right]$ induces the isomorphism $\phi_{1}: E_{2}{ }^{s, s} \rightarrow \tilde{E}_{2}{ }^{s, s}$ by (1) and Proposition 2.4.

Now, for $t-s \geqq 1$, define

$$
\begin{aligned}
\tilde{\beta}_{2}= & j^{s+2} *\left(i_{*}{ }^{(t+1)}\right)^{-1} h^{s+2} *\left(i^{s+1} *\right)^{-1}: D_{2}^{s, t} \longleftrightarrow \mathrm{CGH}_{B}\left[\Sigma_{B}^{t-s} X^{s+1} ; Y\right] \longrightarrow \\
& \mathrm{CGH}_{B}\left[\Sigma_{B}^{t-s-1} X^{s+2} / X^{s+1} ; Y\right]=\mathrm{CGH}_{B}\left[\Sigma_{B}^{t-s-1} X^{s+2} / X^{s+1} ; Y^{(t+1)}\right] \longrightarrow \tilde{E}_{2}^{s+2, t+1}
\end{aligned}
$$

$\tilde{\gamma}_{2}: \tilde{E}_{2}^{s+2, t+1} \subset \mathrm{CGH}_{B}\left[\Sigma_{B}^{t-s-1} X^{s+2} ; Y^{(t+1)}\right] \longrightarrow \mathrm{CGH}_{B}\left[\Sigma_{B}^{t-s-1} X^{s+2} ; Y\right] \supset D_{2}^{s+2, t+1}$.

We shall prove the commutativity of the diagram (3.6). The commutativities of (1), (2), (3) and (5) are obtained by the naturality and the definitions of maps involved. So we omit the proofs.

For the commutativity of (4), consider the diagram

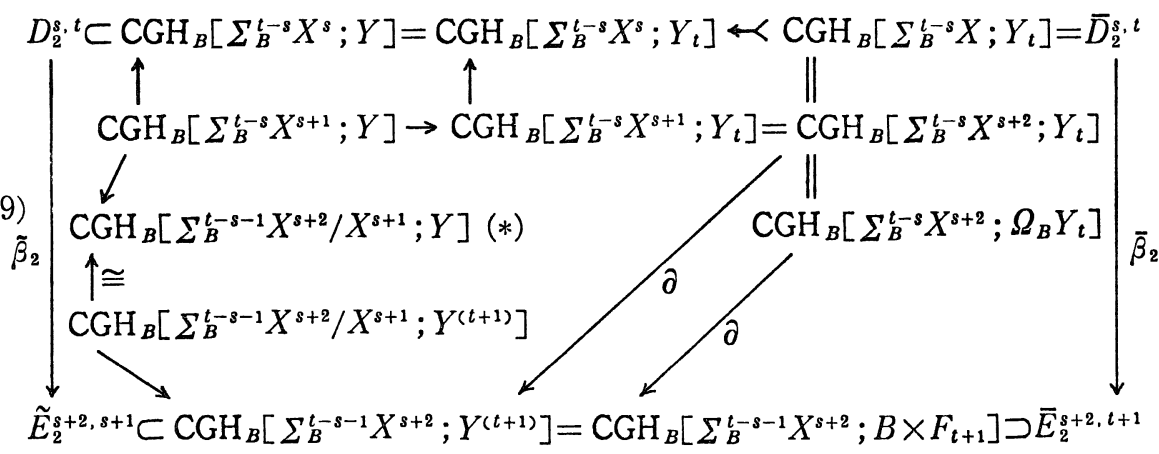

The commutativity of the part $(*)$ is proved by applying Proposition 1.5 to the next diagram.

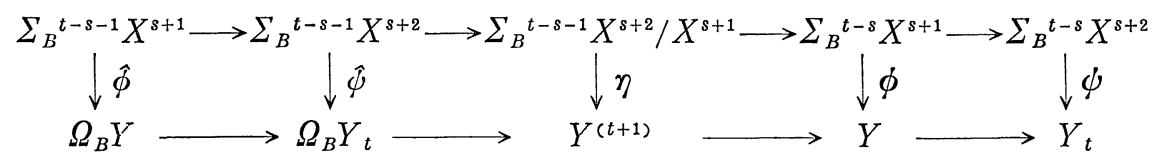

The other commutativities of (3.9) are obtained by the definitions and naturalities. Thus we proved the commutativity of (4). This complete the proof of Theorem 3.5. 
Now we shall investigate the spectral sequence associated with the ex-antiskeleton filtration. By using the Puppe sequence

$$
X^{s} / X^{s-1} \stackrel{f_{s}}{\longrightarrow} X / X^{s-1} \stackrel{g_{s}}{\longrightarrow} X / X^{s} \stackrel{h_{s}}{\longrightarrow} \Sigma_{B} X^{s} / X^{s-1} \longrightarrow \Sigma_{B} X / X^{s-1}
$$

we define the exact couple as follow:

$$
\begin{aligned}
& \left\{D_{1}^{\prime s, t}, E_{1}^{\prime s, t}, \alpha_{1}^{\prime}, \beta_{1}^{\prime}, \gamma_{1}^{\prime}\right\} \\
& D_{1}^{\prime s, t}=\mathrm{CGH}_{B}\left[\Sigma_{B}{ }^{t-s-1} X / X^{s} ; Y\right] \quad(t \geqq s \geqq 0) \\
& E_{1}^{\prime s, t}=\mathrm{CGH}_{B}\left[\Sigma_{B}^{t-s} X^{s} / X^{s-1} ; Y\right] \quad(t \geqq s \geqq 0)
\end{aligned}
$$

where $\alpha_{1}^{\prime}, \beta_{1}^{\prime}, \gamma_{1}^{\prime}$ are induced by $g_{s}: X / X^{s-1} \rightarrow X / X^{s}, f_{s}: X^{s} / X^{s-1} \rightarrow X / X^{s-1}$ and $h_{s}: X / X^{s-1} \rightarrow \Sigma X^{s} / X^{s-1}$ and bidegrees $\alpha_{1}^{\prime}, \beta_{1}^{\prime}, \gamma_{1}^{\prime}$ are $(-1,-1),(1,0),(0,0)$ respectively.

Theorem 3.11. Let $(B \stackrel{y}{\rightarrow} Y \stackrel{q}{\rightarrow} B)$ be an object in $\mathrm{CGH}_{B}$ where $q$ is a fibration with $a$ fiber $F$ and admit a principal Postnikov system. Then there exist a following system called anti-Postnikov system.

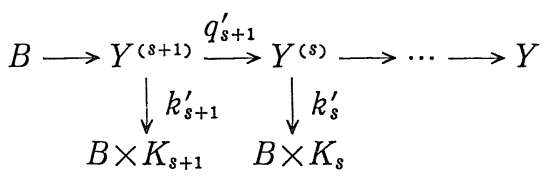

where $Y^{(s+1)}$ is induced by $k_{s}^{\prime}$ from the standard path fibration on $B \times K_{s}$.

Proof. This is clear from Theorem 2.3 and Definition 2.5.

Similarly we can define the spectral sequence associated with the antiPostnikov system (3.12). We define the exact couple:

$$
\begin{array}{ll}
\left\{D_{2}^{\prime \prime s, t}, E_{2}^{\prime \prime s, t}, \alpha_{2}^{\prime \prime}, \beta_{2}^{\prime \prime}, \gamma_{2}^{\prime \prime}\right\} & \\
D_{2}^{\prime \prime s, t}=\mathrm{CGH}_{B}\left[X ; \Omega_{B}{ }^{t-s-1} Y^{(t+1)}\right] & (t \geqq s \geqq 0) \\
E_{2}^{\prime \prime s, t}=\mathrm{CGH}_{B}\left[X ; \Omega_{B}{ }^{t-s}\left(B \times K_{t}\right)\right] & (t \geqq s \geqq 0)
\end{array}
$$

where $\alpha_{2}^{\prime \prime}, \beta_{2}^{\prime \prime}$ and $\gamma_{2}^{\prime \prime}$ are induced by $q_{s+1}^{\prime}: Y^{(s+1)} \rightarrow Y^{(s)}, k_{s}^{\prime}: Y^{(s)} \rightarrow B \times K_{s}$ and $h_{s}^{\prime}: \Omega_{B}\left(B \times K_{s}\right) \rightarrow Y^{(s+1)}$ and bidegrees $\alpha_{2}^{\prime \prime}, \beta_{2}^{\prime \prime}, \gamma_{2}^{\prime \prime}$ are $(-1,-1),(2,1),(0,0)$ respectively.

By using Proposition 2.6, we have the following result by the same way as Theorem 3.5 .

Theorem 3.14. Under the assumptions of Theorem 3.5, there exist natural isomorphisms:

$$
\phi: D_{r}^{\prime s, t} \rightarrow D_{r}^{\prime \prime s, t}, \quad \phi: E_{r}^{\prime s, t} \rightarrow E_{r}^{\prime \prime s, t}
$$

which commute with $\alpha_{r}^{\prime}, \beta_{r}^{\prime}, \gamma_{r}^{\prime}$ and $\alpha_{r}^{\prime \prime}, \beta_{r}^{\prime \prime}, \gamma_{r}^{\prime \prime}$. 


\section{References}

[1] Baues, H. J., Obstruction Theory, Lecture Notes in Math., 628, Springer, 1977.

[2] James, I. M., Ex-homotopy theory 1, Illinois J. Math., 15 (1971), 324-337.

[3] MacLane, S., Categories for working mathematicians, G. T. M., 5, Springer, 1972.

[4] Matumoto, T., Equivariant cohomology theories on G-CW complexes, Osaka J. Math., 10 (1973), 51-68.

[5] Maunder, C.R.F., The Spectral sequence of an extraordinary cohomology, Proc. Cambridge Phil. Soc., 59 (1963), 567-574.

[6] Oda, N. and Shitanda, Y., On the unstable homotopy spectral sequences, Manus. Math., 56 (1986), 19-35.

[7] Shitanda, Y., Abstract homotopy theory and homotopy theory of functor category, to appear in Hiroshima Math. J.

[8] Whitehead, G.W., Elements of Homotopy Theory, G. T. M., 61, Springer, 1978. 
